\title{
Leri-Weill dyschondrosteosis in a newborn presenting with respiratory failure due to severe micrognathia
}

\author{
Mi Hyeon Gang ${ }^{1 \oplus}$, Jianne Lee ${ }^{1 \oplus}$, Yong Wook Lee ${ }^{1 \oplus}$, Ji Hye Shin ${ }^{1 \oplus}$, Han Hyuk Lim ${ }^{1 \oplus}$, Yoo-Mi Kim² ${ }^{\oplus}$, and Mea-young Chang ${ }^{1, *}$ \\ ${ }^{1}$ Department of Pediatrics, Chungnam National University Hospital, Daejeon, Korea \\ ${ }^{2}$ Department of Pediatrics, Chungnam National University Sejong Hospital, Sejong, Korea
}

\begin{abstract}
Short stature homeobox-containing gene $(\mathrm{SHOX})$ is a well-known causative gene for the short stature in Turner syndrome. The clinical manifestation of SHOX gene related disorders varies from SHOX haploinsufficiency, presenting with idiopathic short stature, disproportionate short stature, or Leri-Weill dyschondrosteosis (LWD) to recessive form of extreme dwarfism and limb deformity in Langer mesomelic dysplasia. LWD is usually diagnosed upon suspicion based on short stature and skeletal abnormalities, and it is rarely accompanied with respiratory failure in the neonatal period. Here, we report the case of a newborn infant with LWD presenting with severe micrognathia that caused respiratory distress, which was diagnosed using microarray testing. Even when the manifestation of Madelung deformity is not yet apparent, LWD should be considered as one of underlying diseases related to congenital micrognathia.
\end{abstract}

Key words: SHOX gene, Leri-weill dyschondrosteosis, Micrognathia, Newborn infant.

\section{Introduction}

Haploinsufficiency of short stature homeobox-containing gene (SHOX,NM_000451) in the pseudoautosomal region 1 of $X$ and $Y$ chromosomes can result in a wide spectrum of short stature phenotypes, including Turner syndrome (TS), Leri-Weill dyschondrosteosis (LWD) and short stature without any specific features [1]. SHOX deficiency disorders are inherited in a pseudoautosomal dominant manner.

LWD is characterized by disproportionate short stature, mesomelic limb shortening, and the Madelung deformity of the forearm (bowing of the radius and distal dislocation of the ulna) [2]. Patients with LWD may also exhibit clinical features, such as micrognathia, high-arched palate, and muscular hypertrophy [2]. LWD usually causes short stature, high-arched palate, and characteristic skeletal features in young children [3].

Herein, we report the case of a newborn infant with LWD presenting with severe micrognathia that caused respiratory distress.

\section{Case}

A female infant had been born to a 39-year-old woman. The mother was referred to our hospital for the evaluation of intrauterine fetal growth restriction complicated with oligohydramnios and fetal micrognathia detected on routine fetal sonography at 24 weeks of gestation. Two elder brothers and one elder sister of the female infant were healthy.

The baby was born at a gestational age of 36 weeks and 4 days via cesarean section and weighed 2,000 $\mathrm{g}$ compatible with

\footnotetext{
Received: 13 November 2020, Revised: 20 November 2020, Accepted: 23 November 2020, Published: 31 December 2020

*Corresponding author: Mea-young Chang, M.D., Ph.D. (iD https://orcid.org/0000-0001-5368-3296

Department of Pediatrics, College of Medicine, Chungnam National University Hospital, 282 Munhwa-ro, Jung-gu, Daejeon 35015, Korea.

Tel: +82-42-280-7253, Fax: :+82-42-255-3158, E-mail: mychang@cnuh.co.kr

Conflict of interest: The authors declare that they do not have any conflicts of interest.

(c) This is an open-access article distributed under the terms of the Creative Commons Attribution Non-Commercial License (http://creativecommons.org/licenses/by-nc/4.0/) which permits unrestricted non-commercial use, distribution, and reproduction in any medium, provided the original work is properly cited.

(c) Copyright 2020 by the Korean Society of Medical Genetics and Genomics 
small for gestational age. She was lethargic with a weak cry at birth and needed positive pressure ventilation; the 1- and 5-min Apgar scores were 4 and 7, respectively. She showed marked respiratory difficulty with cyanosis and chest wall retractions. She also had dysmorphic features, including a prominent forehead, low set ears, low nasal bridge, severe micrognathia, retrognathia, cleft soft palate, short neck, and mild ambiguous genitalia with labial hypoplasia and clitoromegaly (Fig. 1). An infantogram showed hemivertebra of S1 and nonspecific findings in both lung fields (Fig. 2A). Nasal continuous positive airway pressure was initially applied, and she was weaned to room air by the third day in the hospital. Pulse oxygen saturation levels were kept relatively stable only if she was placed in a prone position. The growth profiles of birth weight, height, and head circumference were $2 \mathrm{~kg}$ (-2.2 standard deviation score [SDS]), $44 \mathrm{~cm}$ $(-1.45$ SDS), and $32 \mathrm{~cm}(-0.49$ SDS), respectively.

On the day of her birth, complete blood count and serum biochemistry values were normal. The pelvic ultrasonography performed 7 day after birth showed no remarkable findings in the uterus and bilateral ovaries were difficult to delineate. Free thyroxine and thyroid-stimulating hormone levels measured 14 days after birth were $1.47 \mathrm{ng} / \mathrm{dL}$ and $6.36 \mu \mathrm{U} / \mathrm{mL}$, respectively. Other hormone levels were not measured. Chromosome analysis of the patient confirmed 46, XX karyotype, and $947 \mathrm{~Kb}$ deletion at Xp22.33, including SHOX, delineated using chromosomal microarray (Fig. 3). The array comparative genomic hybridization (CGH) analysis was performed using the SurePrint G3 Human CGH Microarray 180K kit (Agilent Technologies, Inc., Santa Clara, CA, USA). Magnetic resonance imaging of the brain demonstrated diffusion restriction foci on the left-side genu of the cor-

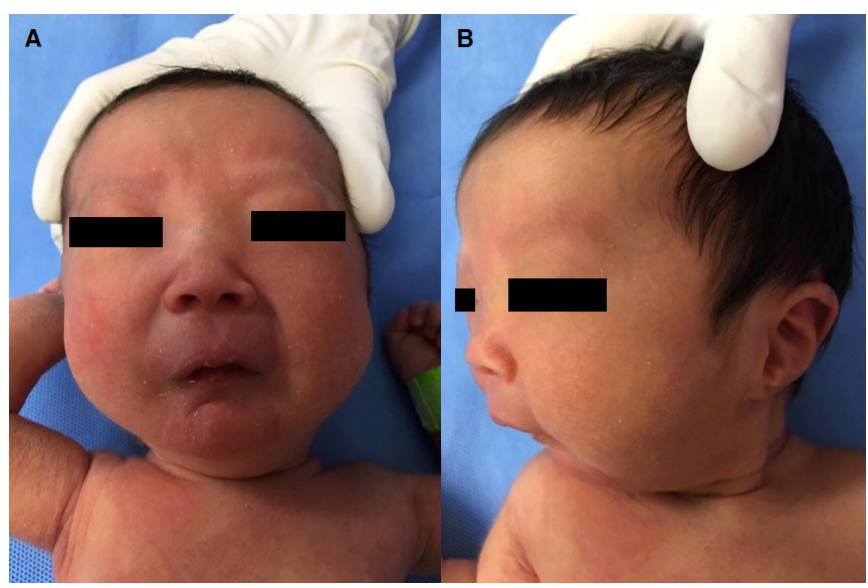

Fig. 1. Clinical phenotypes of the patient showing dysmorphic features including low nasal bridge, low set ear, hypertelorism, severe micrognathia, and retrognathia $(A, B)$. pus callosum, suggesting hypoxic-ischemic injury. Radiographs of the hand, wrist, and forearm showed no remarkable bone abnormality at that time. Hypoplasia of both fibulae was first observed on the leg X-ray taken 53 days after birth (Fig. 2B). An $X$-ray taken at 20 months of age did not show the ossification center of the right femoral head (Fig. 2C). There was no characteristic finding of developmental dysplasia of the hip (DDH) in the hip ultrasonography performed at 10 months of age. She was followed up at the department of orthopedics in another hospital. At the 21-month outpatient follow-up, we received information that she was diagnosed with DDH at the orthopedic department of another hospital and scheduled for surgery in the

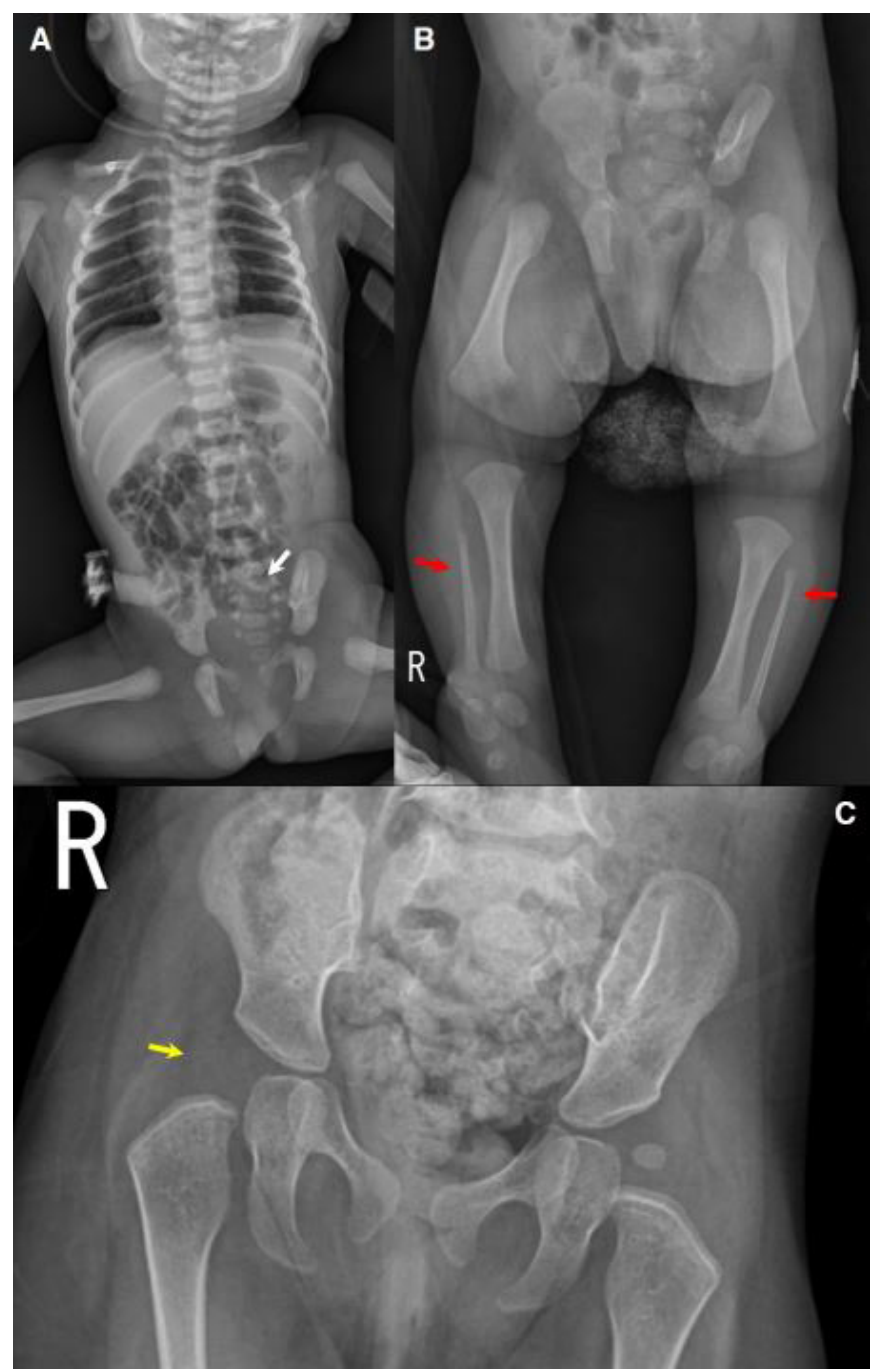

Fig. 2. Radiological findings of the patient. Infantogram of the patient showing hemivertebra of S1 indicated by the white arrow (A). Radiographs of both legs showing hypoplastic fibulae taken 53 days after birth indicated by red arrows (B). No ossification center of the right femoral head visible on a radiograph taken at 20 months of age suggestive of developmental dysplasia of the hip indicated by the yellow arrow (C). 


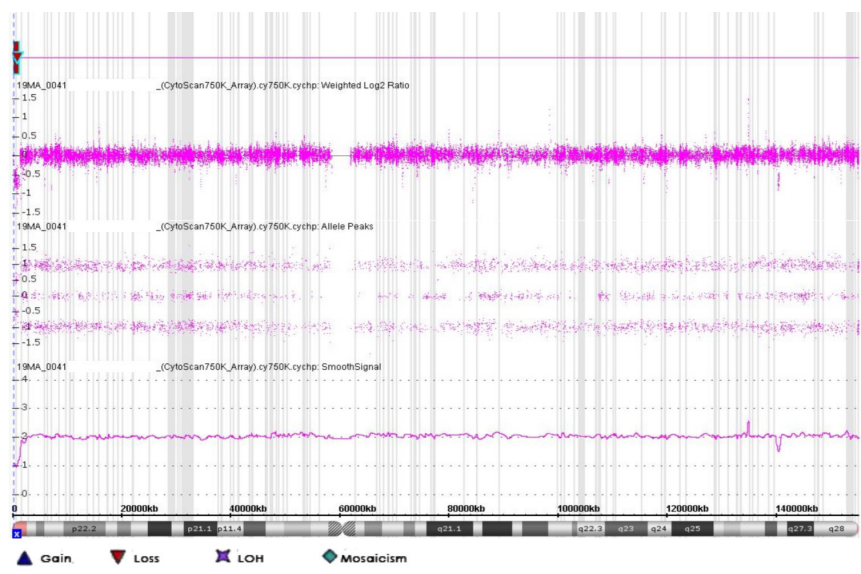

Fig. 3. Chromosomal microarray analysis of the patient. A $947 \mathrm{~Kb}$ deletion at Xp22.33 region 178 from 288066 to 1234634 (Hg19) indicated by a red triangle.

other hospital.

The patient was fed by tube and bottle during hospitalization. On the forty-second day at the hospital, she underwent elective tracheostomy because of persistent airway obstruction secondary to micrognathia. After educating and training caregivers for feeding and tracheostomy care, she was discharged on the seventy-sixth day. The tracheostomy was closed at 3-month of age.

At the 21-month follow-up, she was undergoing rehabilitation treatment because of developmental delay. At this visit, her weight, height, and head circumference were $5.6 \mathrm{~kg}(-6.44 \mathrm{SDS})$, $66 \mathrm{~cm}$ (-5.94 SDS), and $43.3 \mathrm{~cm}(-2.55$ SDS), respectively (Fig. 4). Even though parents showed no skeletal deformity including Madelung deformity or mesomelia, they had familial short stature. The paternal height, maternal height, and mid parental height were $160 \mathrm{~cm}(-2.42$ SDS), $155 \mathrm{~cm}$ (-1.16 SDS), and 151 cm (-2.01 SDS), respectively. However, we could not perform parental genetic testing because of their refusal. Recombinant human growth hormone therapy is planned for this patient.

\section{Discussion}

The SHOX gene was first discovered as the causative gene for growth failure in idiopathic short stature and TS by Rao et al. [4] in 1997.SHOX plays a critical role in chondrocyte development by regulating the cell cycle and apoptosis of hypertrophic chondrocytes. SHOX deficiency leads to perturbed programmed cell death of hypertrophic chondrocytes, which may be related to the skeletal changes [5]. Haploinsufficiency of the SHOX gene is associated with short stature in patients with TS, LWD, and

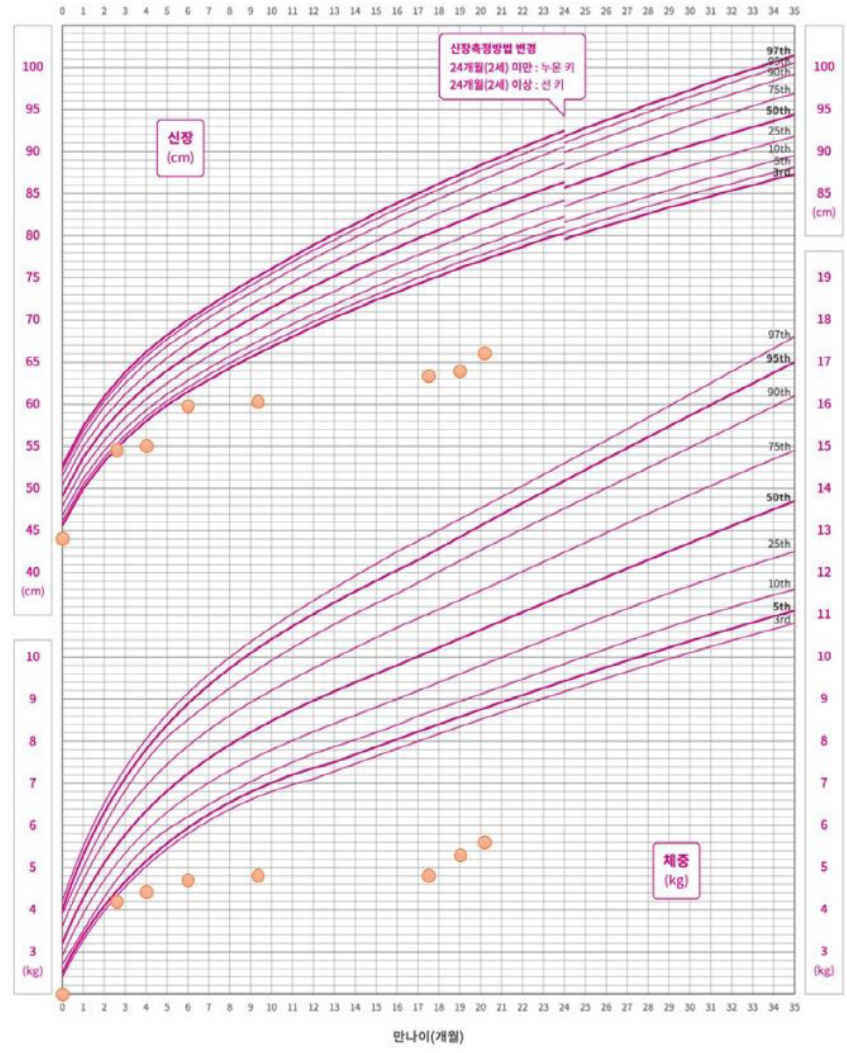

Fig. 4. Growth curve of the patient from birth to 21 months of age.

isolated short stature, and homozygous loss of SHOX induces Langer mesomelic dysplasia [6]. Although TS and LWD have common characteristics of SHOX haploinsufficiency and phenotypic features, such as short stature and skeletal anomalies, TS is distinguished from LWD by ovarian failure and extra-skeletal manifestations [7]. Patients are diagnosed with LWD when Madelung deformity is accompanied by short stature and mesomelic limb shortening [8]. Although LWD is caused by mutations on or near the SHOX gene in around 70\% of the cases, the cause of this disorder remains unknown in those cases not related to the SHOX gene. The major skeletal anomalies related to LWD described above vary according to the time of disease onset, mainly appearing in teens and worsening with puberty [9]. The dysmorphic signs that can appear in LWD other than Madelung deformity and mesomelic short stature include micrognathia, high-arched palate, cubitus valgus, bowing of the forearm and tibia, genu valgum, dislocation of ulna, scoliosis, and muscular hypertrophy [2,5]. According to Rappold et al. [2], 40.6\% of 32 patients with LWD have micrognathia, which is significantly higher than $8.8 \%$ of patients with non-syndromic short stature. However, there have been no reported cases of LWD diagnosed in the neonatal period through micrognathia, which causes 
respiratory failure so far. The patient, in our case, showed fetal growth restriction, life-threatening respiratory failure related to severe micrognathia in the neonatal period, as well as progressive growth failure and skeletal deformities. Although Madelung deformity was not seen during the observation period, mesomelia of the lower extremities was identified from an early age. The ossification center of the right femoral head did not appear until 20 months of age.

In Korea, there are a few reports of pediatric LWD [10-12]. One case report of a clinically diagnosed LWD in an 11-year-old girl showed short stature, Madelung deformity, mesomelic dysplasia and normal karyotype [10]. In 2015, a case of LWD diagnosed using SHOX gene mutation analysis was reported in a 7-yearold girl with short stature and Madelung deformity [11]. A recent study of 23 Korean patients with SHOX haploinsufficiency demonstrated that all patients showed mesomelia and a high proportion of Madelung deformity (56.5\%) [12]. They showed a high proportion of SHOX gene deletion (73.3\%) compared to a point mutation on SHOX, even though there was no genotypephenotype correlation [12].

In summary, while LWD is usually diagnosed during the evaluation of short stature in children or adolescents, we report a case in which LWD was diagnosed using microarray testing because of severe micrognathia, causing life-threatening respiratory failure in the neonatal period. Even when the manifestation of Madelung deformity is not yet apparent, LWD should be considered as one of the underlying diseases related to congenital micrognathia.

\section{Acknowledgements}

We thank our patient and her family members for their participation in this study.

\section{References}

1. Jorge AA, Funari MF, Nishi MY, Mendonca BB. Short stature caused by isolated SHOX gene haploinsufficiency: update on the diagnosis and treatment. Pediatr Endocrinol Rev 2010;8:79-85.

2. Rappold G, Blum WF, Shavrikova EP, Crowe BJ, Roeth R, Quigley CA, et al. Genotypes and phenotypes in children with short stature: clinical indicators of SHOX haploinsufficiency. J Med Genet 2007;44:30613.

3. Ross JL, Kowal K, Quigley CA, Blum WF, Cutler GB Jr, Crowe B, et al. The phenotype of short stature homeobox gene (SHOX) deficiency in childhood: contrasting children with Leri-Weill dyschondrosteosis and Turner syndrome. J Pediatr 2005;147:499-507.

4. Rao E, Weiss B, Fukami M, Rump A, Niesler B, Mertz A, et al. Pseudoautosomal deletions encompassing a novel homeobox gene cause growth failure in idiopathic short stature and Turner syndrome. Nat Genet 1997;16:54-63.

5. Seki $A$, Jinno $T$, Suzuki E, Takayama S, Ogata T, Fukami M. Skeletal deformity associated with SHOX deficiency. Clin Pediatr Endocrinol 2014;23:65-72.

6. Jorge AA, Souza SC, Nishi MY, Billerbeck AE, Libório DC, Kim CA, et al. SHOX mutations in idiopathic short stature and Leri-Weill dyschondrosteosis: frequency and phenotypic variability. Clin Endocrinol (Oxf) 2007:66:130-5.

7. Ross JL, Scott C Jr, Marttila P, Kowal K, Nass A, Papenhausen $P$, et al. Phenotypes associated with SHOX deficiency. J Clin Endocrinol Metab 2001;86:5674-80.

8. Binder G. Short stature due to SHOX deficiency: genotype, phenotype, and therapy. Horm Res Paediatr 2011;75:81-9.

9. Depeyre A, Schlund M, Nicot R, Ferri J. Dental and maxillofacial signs in Leri-Weill dyschondrosteosis. J Oral Maxillofac Surg 2019;77:7628.

10. Eun $\mathrm{SE}$, Park $H J$, Jung $M H$, Lee BC. A case of Leri-Weill syndrome. J Korean Soc Pediatr Endocrinol 2001;6:165-70.

11. Choi WB, Seo SH, Yoo WH, Kim SY, Kwak MJ. A Leri-Weill dyschondrosteosis patient confirmed by mutation analysis of SHOX gene. Ann Pediatr Endocrinol Metab 2015;20:162-5.

12. Lee JS, Kim HY, Lee YA, Lee SY, Cho TJ, Ko JM. Clinical and genetic characteristics of 23 Korean patients with haploinsufficiency of the short-stature homeobox-containing gene. Exp Clin Endocrinol Diabetes 2020, in press. 